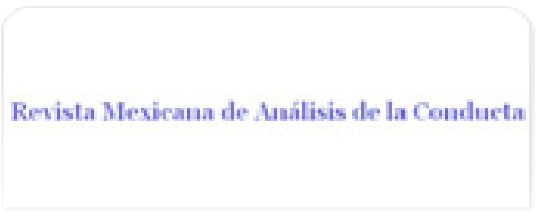

Revista Mexicana de Análisis de la Conducta ISSN: 0185-4534

editora@rmac-mx.org

Sociedad Mexicana de Análisis de la Conducta México

Galindo, Alma; López-Espinoza, Antonio

Efectos del sabor y del contenido calórico del agua sobre la conducta alimentaria durante un período de privación de comida en ratas albinas

Revista Mexicana de Análisis de la Conducta, vol. 32, núm. 2, diciembre, 2006, pp. 95-109

Sociedad Mexicana de Análisis de la Conducta

Guadalajara, México

Disponible en: http://www.redalyc.org/articulo.oa?id=59332202

Cómo citar el artículo

- Número completo

- Más información del artículo

Página de la revista en redalyc.org

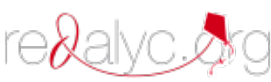

Sistema de Información Científica

Red de Revistas Científicas de América Latina, el Caribe, España y Portugal Proyecto académico sin fines de lucro, desarrollado bajo la iniciativa de acceso abierto 


\title{
EFECTOS DEL SABOR Y DEL CONTENIDO CALÓRICO DEL AGUA SOBRE LA CONDUCTA ALIMENTARIA DURANTE UN PERÍODO DE PRIVACIÓN DE COMIDA EN RATAS ALBINAS
}

\author{
EFFECTS OF FLAVOR AND CALORIC CONTENT OF WATER ON \\ FEEDING BEHAVIOR DURING \\ FOOD DEPRIVATION PERIOD IN ALBINO RATS
}

\author{
ALMA GALINDO Y ANTONIO LÓPEZ-ESPINOZA ${ }^{1}$ \\ UNIVERSIDAD DE GUADALAJARA
}

\begin{abstract}
RESUMEN
Doce ratas Wistar fueron asignadas a dos grupos experimentales y uno control, cada grupo con dos hembras y dos machos. Se inició el experimento con 10 días de libre acceso; posteriormente, el Grupo 1 mantuvo disponible una concentración de azúcar y el Grupo 2 una de quinina, ambas diluidas en agua, durante 5 días. Después retornaron a libre acceso por 10 días. Posteriormente, el Grupo 1 se expuso a la concentración de quinina, y el Grupo 2 a la de azúcar durante 5 días, regresando a libre acceso por 10 días. A continuación, ambos grupos fueron expuestos a una mezcla de azúcar y quinina diluidos en agua durante 5 días, seguidos de 10 días de libre acceso. Finalmente, fueron privados de comida y expuestos a la mezcla agua-glucosa-quinina durante 8 días, finalizando con 10 días de libre acceso. El grupo control se mantuvo en libre acceso todo el experimento. Los resultados indican que el azúcar fue aceptada; contrariamente la quinina y la mezcla fueron rechazadas. Esto

1. Esta investigación fue financiada por el Proyecto 46083-H del Conacyt.

Para correspondencia dirigirse a: Alma Galindo e-mail: almkary@yahoo.com.mx. Antonio López-Espinoza e-mail: anton779@megared.net.mx, Centro de Estudios e Investigaciones en Comportamiento. Universidad de Guadalajara, Francisco de Quevedo núm. 180, Arcos Vallarta, C.P. 44100, Guadalajara, Jalisco, México.
\end{abstract}


sugiere que el sabor es factor determinante para el consumo de la comida, incluso de mayor relevancia que el contenido calórico.

Palabras clave: sabor, contenido calórico, privación de comida, conducta alimentaria, ratas.

\begin{abstract}
Twelve Wistar rats were randomly divided into three groups (two experimental and one control) each one with two females and two males. The experiment began with 10 days of free access; subsequently, group 1 maintained available a concentration of sugar and Group 2 a concentration of quinine, both diluted on water, during 5 days. Immediately both groups returned to free access for 10 days. Immediately, Group 1 was exposed to quinine concentration, and Group 2 to sugar concentration during 5 days, returning to free access for 10 days. Subsequently, both groups were exposed to a mixture of sugar, quinine and water during 5 days, followed by 10 days of free access. Finally, experimental groups were deprived of food and exposed to the mixture of water-glucose-quinine during 8 days, concluding with 10 days of free access. Control group had free access during all the experiment. Results indicate that sugar was accepted; contrarily quinine and combination were rejected. This suggests that flavor is determinant for food consumption, even of greater importance that caloric content.

Key Words: Flavor, caloric content, food deprivation, feeding behavior, rats.
\end{abstract}

El sabor y el contenido calórico del alimento han sido señalados como factores que determinan la elección de la comida. Por un lado, se ha demostrado que el sabor es un factor determinante para la regulación alimentaria, ya que junto con el olor, el color y la textura proporciona al sujeto una serie de señales que determinan la palatabilidad y contenido calórico de la comida (Aubert, 2005; Capaldi, 1996; Sclafani, 1990). Sclafani (1990) afirmó que los organismos responderán a estas señales con base en las preferencias innatas o propias de la especie, y en aquéllas aprendidas a lo largo de su vida. Bartoshuk (1990) y Mennella y Beauchamp (1996) clasificaron la percepción del sabor a partir de cuatro sabores básicos: dulce, salado, amargo y agrio. Se ha documentado que el sabor dulce es preferido sobre los otros sabores por numerosas especies de animales, incluidas las ratas; mientras que los sabores amargo y ácido son evitados (Bartoshuk, 1990; Booth, 1990; Fedorchak y Bolles, 1987; Schafe, 1996).

Mennella y Beauchamp (1996) reportaron, en una serie de estudios rea- 
lizados con humanos, que la experiencia con un sabor es determinante en la elección de la comida. Así, los infantes que estuvieron en contacto con ciertas sustancias a través de la lactancia prefirieron alimentos con sabores similares a los de las sustancias contenidas en la leche materna. Sin embargo, en algunas ocasiones la elección de la comida puede ser contraria a las necesidades metabólicas. Mason (1965) realizó un experimento en el que se utilizaron soluciones de acetato de plomo en varias concentraciones; las ratas consumieron grandes cantidades de las soluciones de baja concentración, mientras que rechazaron las de alta concentración. Es necesario mencionar que el acetato de plomo es una sal venenosa altamente soluble, dulce y "definitivamente aceptable". Los resultados obtenidos demostraron que el sabor es un factor preponderante en la elección de la comida. Esto sustentado en la preferencia mostrada por el sabor dulce a pesar de sus efectos venenosos (Mason, 1965).

Por otro lado, se ha demostrado que el contenido calórico es un factor determinante en la elección de la comida, y que por lo general los alimentos con alto contenido calórico presentan sabores que son aceptados (Bartoshuk, 1990; Booth, 1990; Fedorchak y Bolles, 1987; Schafe, 1996). Rozin (1965) demostró que se puede inducir el apetito por un alimento específico al crear un estado de déficit en el organismo. Ante dos fuentes de alimento (una con tiamina, y la otra sin ella), las ratas privadas de tiamina prefirieron la comida que la contenía, aún después de recuperarse del déficit por la tiamina. Concluyó que la preferencia por algún alimento se encuentra determinada por los efectos postingestivos de la comida, así como por experiencias de privación de agua o comida.

Adicionalmente al sabor y al contenido calórico de la comida, un factor determinante de las conductas de selección y consumo es el sexo de los sujetos. Sclafani y Gorman (1977), realizaron un estudio en el que expusieron a ratas de ambos sexos a dos tipos de dieta: una dieta balanceada para animales de laboratorio, y una dieta de "supermercado", que consistía en galletas de chocolate, bocadillos de queso, cereal cubierto con azúcar, cacahuates y leche condensada azucarada. El efecto de la dieta de "supermercado" fue más notorio en el peso corporal de las hembras que en el de los machos: durante los primeros sesenta días de exposición a la dieta de "supermercado" las hembras aumentaron su peso en un $170 \%$, comparadas con el grupo control, mientras que los machos solamente aumentaron su peso en un $32 \%$. Por otro lado, Martínez (2005) reportó que las ratas hembras consumen mayores cantidades de azúcar diluida en agua que los machos. Debido a que en estos estudios se ha demostrado la importancia del sexo en el consumo de la comida, se consideró necesario, en el presente estudio, incluir hembras y machos en el diseño experimental.

Por otra parte, cuando los organismos se someten a un período de pri- 
vación de agua o de comida, ya sea total o parcial, tienden a modificar el patrón alimentario establecido, y esta modificación tiene consecuencias en la conducta alimentaria aún después de que el período de privación de comida o agua finalizó. Estos efectos incluyen almacenamiento, selección de la comida y los llamados efectos post-privación, que incluyen la recuperación del peso corporal, el aumento del consumo de la comida (o gran comilona) y el aumento del consumo de agua (o gran bebida) (Finger, 1951; López-Espinoza, 2001, 2004; López-Espinoza y Martínez, 2001). Durante la privación de agua o comida, el organismo sufre un déficit que debe ser compensado a través de la modificación de los patrones de conducta. Por lo tanto, buscará nuevas fuentes nutricias que le aporten la energía y sustancias necesarias para compensar el déficit y sobrevivir (Del Prete, 1994).

Factores como el sabor o el contenido calórico determinarán en ciertas circunstancias el consumo o evitación de la comida, estableciendo la modificación de la conducta alimentaria en el organismo. Forestell y LoLordo (2000), en un experimento realizado con ratas, formaron cuatro grupos de sujetos experimentales que fueron expuestos a concentraciones de ácido cítrico o de octaacetato de sucrosa (sustancias con sabores no aceptados por las ratas) mezcladas con agua o con sacarina. Cada grupo fue expuesto a una mezcla de agua con glucosa una hora o tres minutos antes de la presentación de los sabores no aceptados. Las ratas que estuvieron expuestas a los sabores no aceptados mezclados con sacarina bebieron más líquido en comparación con las ratas que fueron expuestas a los sabores no aceptados mezclados solamente con agua. Esto indica que las ratas pueden adquirir preferencias condicionadas por sabores relativamente inaceptables cuando son mezclados con sacarina, lo cual sugiere que las ratas podrían consumir un alimento con sabor no aceptado y, que al ser rico en calorías o estar asociado con ellas, puede ser preferido sobre otras fuentes de alimento. En el presente estudio, los sujetos experimentales fueron expuestos a una sustancia con contenido energético pero con sabor no aceptado durante un estado de privación de comida.

\section{MÉTODO}

\section{Sujetos}

Doce ratas de la cepa Wistar, seis hembras $(\mathrm{H} 1, \mathrm{H} 2, \mathrm{H} 3, \mathrm{H} 4, \mathrm{H} 5$ y $\mathrm{H} 6)$ y seis machos (M1, M2, M3, M4, M5 y M6) experimentalmente ingenuas y con una edad de tres meses al inicio del experimento. 


\section{Aparatos y Materiales}

Se utilizaron doce cajas-habitación individuales, con medidas de $13 \mathrm{~cm}$ de altura por $27 \mathrm{~cm}$ de ancho y $38 \mathrm{~cm}$ de largo, con una reja metálica en la parte superior, con división para comederos y bebederos. El fondo de la caja se mantuvo cubierto por una alfombra de aserrín, que fue removida y substituida por otra cada 4 días. Para el registro del consumo de comida y peso corporal se utilizó una báscula electrónica de precisión. Croquetas de la marca comercial Nutri-cubos, especial para animales de laboratorio, fue el alimento proporcionado. Su fórmula nutricional fue la siguiente: $3 \%$ de grasas, $7 \%$ de cenizas, $1 \%$ de calcio, $23 \%$ de proteína, $6 \%$ de fibra, $49 \%$ de E.L.N. (extracto libre de nitrógeno), $6 \%$ de fósforo y $12 \%$ de humedad. Durante el experimento se proporcionaron $50 \mathrm{~g}$ de nutricubos a cada sujeto, a excepción de los períodos de privación de comida. Como bebida se utilizó agua y durante la manipulación experimental se proporcionó: 1) una solución compuesta de 4 $\mathrm{mg}$ de sulfato de quinina diluida en $200 \mathrm{ml}$ de agua que aportó 0 calorías; 2) una solución compuesta de $30 \mathrm{~g}$ de glucosa diluida en $200 \mathrm{ml}$ de agua que aportó 60 calorías en total; y, 3) una solución compuesta de $4 \mathrm{mg}$ de sulfato de quinina y de $30 \mathrm{~g}$ de glucosa diluidas en $200 \mathrm{ml}$ de agua que aportó 60 calorías en total. El líquido se proporcionó en bebederos graduados de $200 \mathrm{ml}$.

\section{Procedimiento}

Los sujetos fueron identificados con un número de registro, fecha de nacimiento y peso corporal al inicio del experimento. Fueron colocados en cajashabitación individuales, que permanecían en el bioterio con un ciclo de luzobscuridad de $12 \times 12$ horas, a una temperatura promedio de $22^{\circ} \mathrm{C}$. El peso corporal, el consumo de agua y comida se registraron diariamente a las 9:00 de la mañana.

Para calcular las calorías consumidas en el agua con glucosa y en la comida se determinó el número de calorías que aporta la comida por gramo a partir de la media estandarizada en nutrición animal (Subcommittee on Laboratory Animal Nutrition, Committee of Animal Nutrition, Board on Agriculture, National Council, 1995) de 3.4 calorías por gramo consumido. Esta cantidad se multiplicó por el número de gramos consumidos diariamente por el animal. El agua, así como la solución de quinina proporcionaron 0 calorías. Cuando se adicionó glucosa al agua, se calculó el número de calorías que aporta la concentración de glucosa, el cual se multiplicó por el número de mililitros consumidos diariamente. Se sumó la cantidad de calorías consumidas en la comida más el número de calorías consumidas en la bebida para obtener el consumo total diario de calorías.

Se formaron dos grupos de sujetos experimentales y uno control, cada 
grupo se integró por dos hembras y dos machos. Los sujetos M1, M2, H1, y H2 fueron asignados al Grupo 1, los sujetos M3, M4, H3, y H4 al Grupo 2 y los sujetos M5, M6, H5 y H6 se asignaron al Grupo Control. La asignación de todos los sujetos a los grupos experimentales se realizó de forma aleatoria (Tabla 1).

Tabla 1. Muestra el diseño experimental. Las fases 1, 3, 5, 7 y 9 muestran los períodos de libre acceso a la comida y al agua para los Grupos 1 y 2. En las fases 2 y 4 se indica, con dos tonos de gris, la secuencia de exposición (diseñada con el objetivo de valorar y controlar los efectos de la historia de exposición) a la glucosa sin privación de comida y a la quinina sin privación de comida de ambos grupos. En las fases 6 y 8 se indican los períodos de exposición a la mezcla glucosa-quinina sin privación de comida y con privación de comida, respectivamente. El Grupo Control se mantuvo en libre acceso a la comida y al agua durante las nueve fases del experimento.

\begin{tabular}{|c|c|c|c|c|c|c|c|c|c|}
\hline & Fase 1 & Fase 2 & Fase 3 & Fase 4 & Fase 5 & Fase 6 & Fase 7 & Fase 8 & Fase 9 \\
\hline Grupo 1 & $\begin{array}{c}\text { Libre } \\
\text { acceso } \\
\text { a comida } \\
\text { y agua }\end{array}$ & Glucosa & $\begin{array}{c}\text { Libre } \\
\text { acceso } \\
\text { a comida } \\
\text { y agua }\end{array}$ & Quinina & $\begin{array}{c}\text { Libre } \\
\text { acceso } \\
\text { a comida } \\
\text { y agua }\end{array}$ & $\begin{array}{l}\text { Glucosa } \\
\text {-quinina }\end{array}$ & $\begin{array}{c}\text { Libre } \\
\text { acceso } \\
\text { a comida } \\
\text { y agua }\end{array}$ & $\begin{array}{l}\text { Privación- } \\
\text { glucosa- } \\
\text { quinina }\end{array}$ & $\begin{array}{c}\text { Libre } \\
\text { acceso } \\
\text { a comida } \\
\text { y agua }\end{array}$ \\
\hline Grupo 2 & $\begin{array}{c}\text { Libre } \\
\text { acceso } \\
\text { a comida } \\
\text { y agua }\end{array}$ & Quinina & $\begin{array}{l}\text { Libre } \\
\text { acceso } \\
\text { a comida } \\
\text { y agua }\end{array}$ & Glucosa & $\begin{array}{l}\text { Libre } \\
\text { acceso } \\
\text { a comida } \\
\text { y agua }\end{array}$ & $\begin{array}{l}\text { Glucosa } \\
\text {-quinina }\end{array}$ & $\begin{array}{l}\text { Libre } \\
\text { acceso } \\
\text { a comida } \\
\text { y agua }\end{array}$ & $\begin{array}{l}\text { Privación- } \\
\text { glucosa- } \\
\text { quinina }\end{array}$ & $\begin{array}{c}\text { Libre } \\
\text { acceso } \\
\text { a comida } \\
\text { y agua }\end{array}$ \\
\hline $\begin{array}{l}\text { Grupo } \\
\text { Control }\end{array}$ & \multicolumn{9}{|c|}{ Libre acceso } \\
\hline Duración & 10 & 5 & 10 & 5 & 10 & 5 & 10 & 8 & 10 \\
\hline \multicolumn{10}{|c|}{ Días } \\
\hline
\end{tabular}

\section{RESULTADOS}

Las Figuras 1, 2 y 3 muestran los promedios del peso corporal, del consumo de comida y del consumo de agua, respectivamente. Los paneles superiores muestran los datos del Grupo 1, los centrales el Grupo 2 y los inferiores el Grupo Control. Las columnas de la izquierda representan a las hembras y las columnas de la derecha a los machos. La línea continua representa los períodos de libre acceso, libres de quinina y de glucosa, los círculos vacíos el período de exposición a la glucosa, los círculos llenos el período de exposición a la quinina, los triángulos vacíos el período de exposición a la mezcla sin privación de comida y los triángulos llenos el período de exposición a la mezcla con privación de comida.

En la Figura 1 puede observarse que el Grupo 1 y el Grupo 2 muestran 
el mismo patrón de cambios en el peso corporal durante todo el experimento. Esto a pesar de la diferencia en la secuencia experimental entre los dos grupos. Durante la exposición a la quinina, a la mezcla sin privación de comida y a la mezcla con privación de comida, el peso disminuyó gradualmente en un promedio de 33 gramos en las hembras y de 43 gramos en los machos con respecto a los períodos de libre acceso (exposición a nutricubos y agua), mientras que durante la exposición a la glucosa el peso se mantuvo estable tanto en las hembras como en los machos. En los períodos de libre acceso posteriores a la exposición a la quinina, a la mezcla con privación de comida y a la mezcla sin privación de comida se observa la recuperación gradual del peso. El Grupo Control presentó un patrón ascendente en la curva de crecimiento.

Las gráficas de la Figura 2 muestran que el consumo de comida disminuyó durante los períodos de exposición a la glucosa, a la quinina y a la mezcla sin privación de comida en los Grupos 1 y 2 . Durante la exposición a la glucosa las hembras de ambos grupos redujeron el consumo de comida en un promedio de 8 gramos, mientras que los machos lo redujeron en un promedio de 9 gramos, en comparación con los consumos de libre acceso. Durante la exposición a la quinina la reducción en el consumo de comida fue gradual en ambos grupos, hasta casi alcanzar los 0 gramos. Durante la exposición a la mezcla sin privación de comida las hembras de ambos grupos disminuyeron el consumo de comida en un promedio de 11 gramos, mientras que los machos lo redujeron en un promedio de 15 gramos con respecto a los consumos de libre acceso. Durante el período de exposición a la mezcla con privación de comida el consumo de comida fue de 0 en hembras y machos de ambos grupos. Puede observarse que en ambos grupos se presenta un aumento en el consumo de comida (gran comilona), de aproximadamente 5 gramos en las hembras y de 3 gramos en los machos, posteriormente a los períodos de exposición a la quinina, a la mezcla sin privación de comida y a la mezcla con privación de comida. El Grupo Control mantuvo un consumo constante durante todo el experimento: las hembras consumieron un promedio de 19.9 $\mathrm{g}$ con una varianza de $+/-3.2 \mathrm{~g}$ y los machos consumieron un promedio de $26 \mathrm{~g}$ con una varianza de $+/-3.7 \mathrm{~g}$.

Las gráficas de la Figura 3 muestran el consumo de agua. Durante la exposición a la glucosa los Grupos 1 y 2 incrementaron el consumo de agua: las hembras consumieron un promedio de $69 \mathrm{ml}$ y los machos un promedio de 70 $\mathrm{ml}$. Se observa que el consumo de agua disminuyó durante los períodos de exposición a la quinina, a la mezcla sin privación de comida y a la mezcla con privación de comida: durante la exposición a la quinina las hembras consumieron un promedio de $13 \mathrm{ml}$ y los machos un promedio de $15 \mathrm{ml}$; durante la exposición a la mezcla sin privación de comida las hembras consumieron un promedio de $11 \mathrm{ml}$ y los machos un promedio de $8 \mathrm{ml}$; y durante la exposición 


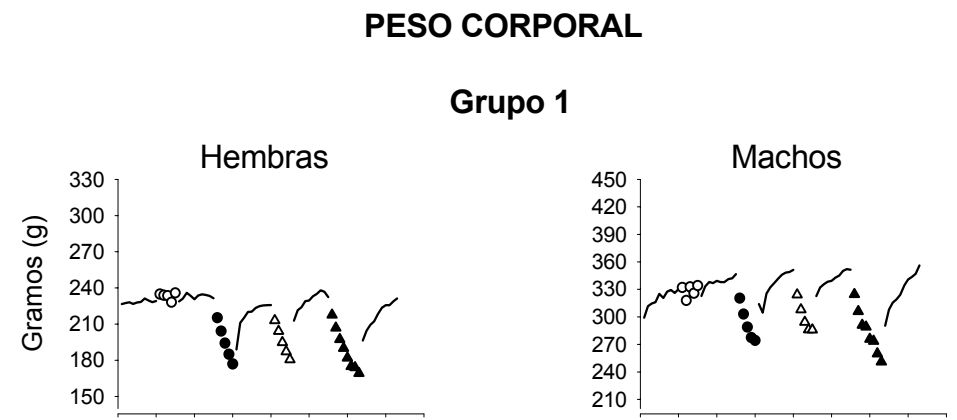

\section{Grupo 2}
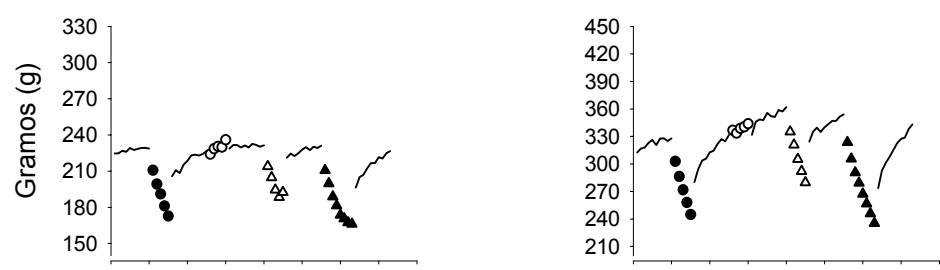

\section{Grupo Control}
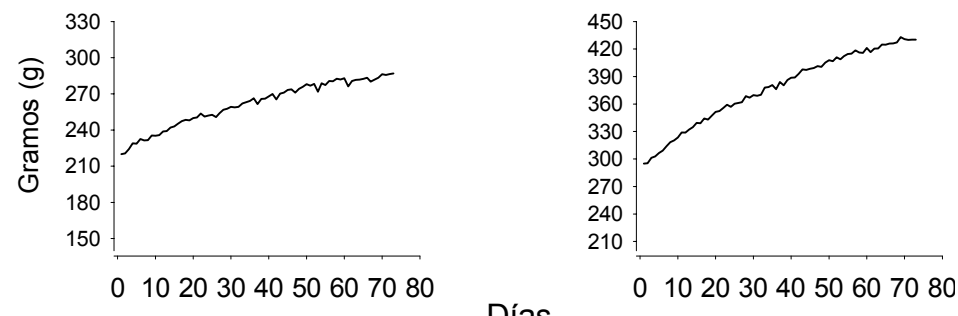

Figura 1. Muestra los promedios del peso corporal del Grupo 1, del Grupo 2 y del Grupo Control, de hembras y machos. Las líneas continuas representan el libre acceso, los círculos vacíos el período de exposición a la glucosa, los círculos llenos el período de exposición a la quinina, los triángulos vacíos el período de exposición a la mezcla sin privación de comida y los triángulos llenos el período de exposición a la mezcla con privación de comida. 


\section{CONSUMO DE ALIMENTO}

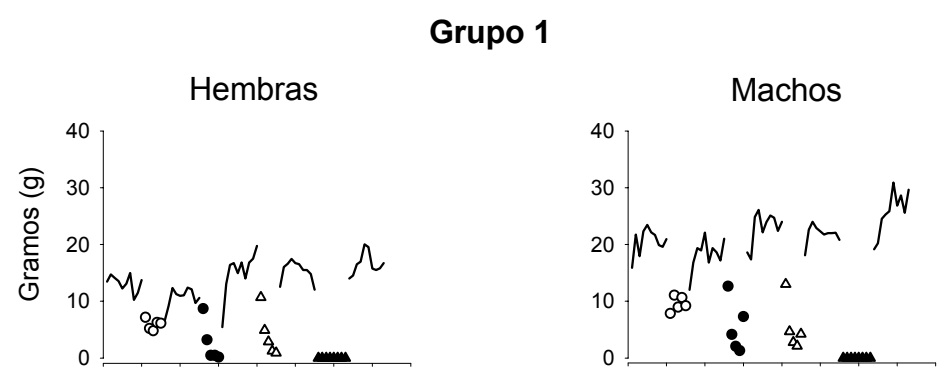

\section{Grupo 2}
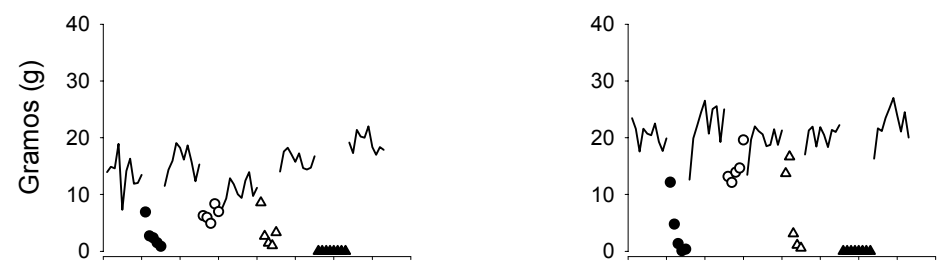

\section{Grupo Control}
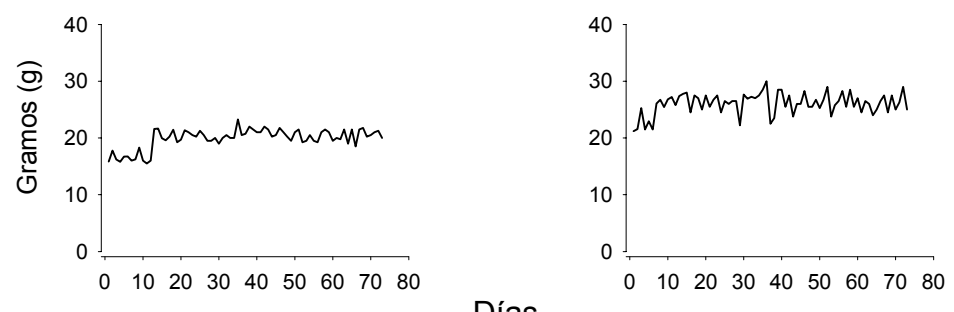

Figura 2. Muestra los promedios del consumo de comida del Grupo 1, del Grupo 2 y del Grupo Control, de hembras y machos. Las líneas continuas representan el libre acceso, los círculos vacíos el período de exposición a la glucosa, los círculos llenos el período de exposición a la quinina, los triángulos vacíos el período de exposición a la mezcla sin privación de comida y los triángulos llenos el período de exposición a la mezcla con privación de comida. 
a la mezcla con privación de comida las hembras consumieron un promedio de $22 \mathrm{ml}$ y los machos un promedio de $14 \mathrm{ml}$. Se presentaron grandes bebidas, en promedio las hembras consumieron $53 \mathrm{ml}$ y los machos $72 \mathrm{ml}$. En el Grupo Control, tanto las hembras como los machos consumieron un promedio de $52 \mathrm{ml}$ durante todo el experimento.

Finalmente, la Figura 4 muestra los promedios de las calorías consumidas por hembras y machos de los Grupos 1, 2 y Control durante todo el experimento. Las barras rayadas indican el total de calorías obtenidas de los nutricubos, las barras grises el total de calorías obtenidas de la glucosa; las barras negras indican las calorías obtenidas de los nutricubos durante la exposición a la solución de quinina, y las barras blancas muestran el total de calorías obtenidas de la mezcla glucosa-quinina. Se puede observar que el número de calorías obtenidas de la glucosa es superior o similar al número de calorías obtenidas de los nutricubos durante la exposición a la glucosa en los Grupos 1 y 2. Asimismo, el número de calorías obtenidas de nutricubos durante la exposición a la quinina disminuyó en ambos grupos.

Durante la exposición a la mezcla glucosa-quinina sin privación de comida, las calorías obtenidas de nutricubos disminuyeron de forma semejante en los Grupos 1 y 2. Las calorías provenientes de la mezcla fueron mínimas en ambos grupos, con excepción de las calorías obtenidas por las hembras del Grupo 2, que consumieron más calorías a través de la mezcla que de los nutricubos. Durante la exposición a la mezcla glucosa-quinina con privación de comida, el promedio de calorías obtenidas de la mezcla por los Grupos 1 y 2 fue de 11, mayor al número de calorías obtenidas de la mezcla durante la exposición a la mezcla glucosa-quinina sin privación de comida, con un promedio de 7 calorías; adicionalmente, durante la exposición a la mezcla glucosa-quinina con privación de comida, las hembras del Grupo 2 consumieron mayor cantidad de calorías (en promedio 19.5) provenientes de la mezcla que el resto de los sujetos.

El total de calorías obtenidas de nutricubos durante los períodos de libre acceso se mantuvo relativamente estable, no observándose ninguna diferencia importante entre ambos grupos. El total de calorías obtenidas de nutricubos por el Grupo Control se mantuvo estable durante todo el experimento.

\section{DISCUSIÓN}

Los resultados obtenidos mostraron que los sujetos experimentales: 1) aumentaron el consumo de agua y disminuyeron el consumo de comida durante la fase de exposición a la glucosa; 2) disminuyeron el peso corporal, el consumo de comida y de agua durante la fase de exposición a la quinina; 3) disminuyeron el peso, el consumo de agua y comida durante la fase de 


\section{CONSUMO DE AGUA}

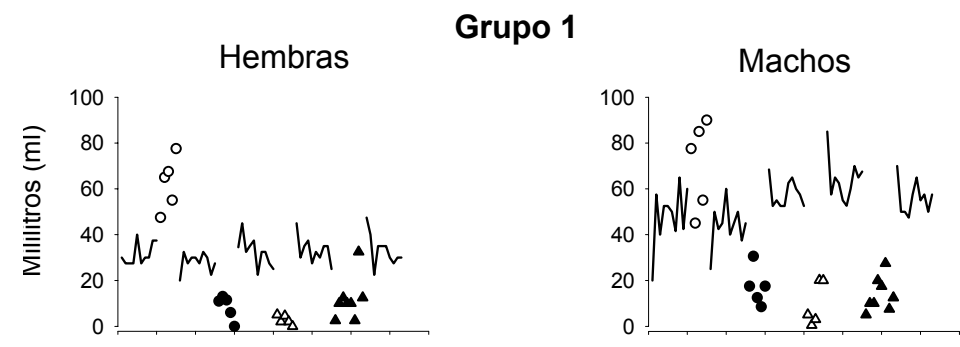

\section{Grupo 2}
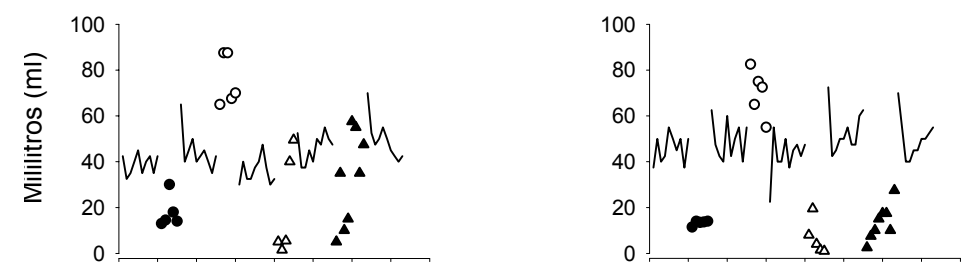

\section{Grupo Control}
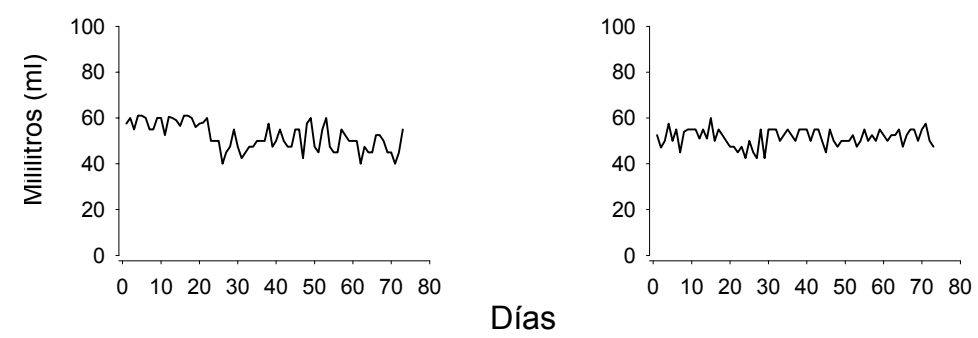

Figura 3. Muestra los promedios del consumo de agua del Grupo 1, del Grupo 2 y del Grupo Control, de hembras y machos. Las líneas continuas representan el libre acceso, los círculos vacíos el período de exposición a la glucosa, los círculos llenos el período de exposición a la quinina, los triángulos vacíos el período de exposición a la mezcla sin privación de comida y los triángulos llenos el período de exposición a la mezcla con privación de comida. 
PROMEDIO DE CALORIAS CONSUMIDAS
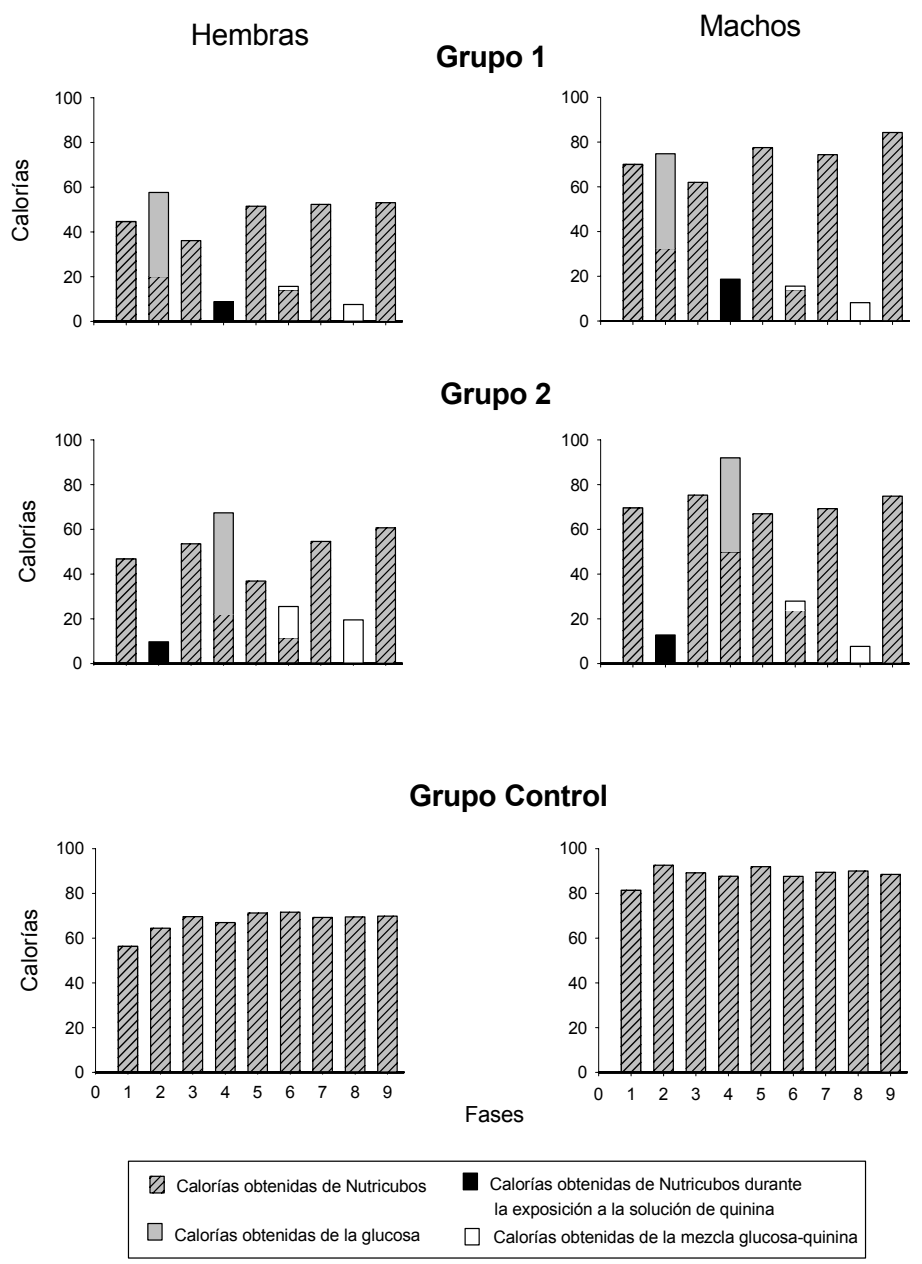

Figura 4. Promedios del consumo de calorías de hembras y machos de los Grupos 1, 2 y Control durante las 9 fases del experimento. Las barras rayadas indican el total de calorías obtenidas de nutricubos, las barras grises el total de calorías obtenidas de la glucosa; las barras negras indican las calorías obtenidas de nutricubos durante la exposición a la solución de quinina, y las barras blancas muestran el total de calorías obtenidas de la mezcla glucosa-quinina. 
exposición a la mezcla sin privación de comida; y, 4) disminuyeron el peso y el consumo de agua durante la fase de exposición a la mezcla con privación de comida. Los sujetos control mantuvieron el peso corporal, consumo de comida y agua estables durante todo el experimento.

Los datos obtenidos sugieren que el sabor de la quinina no fue aceptado, aún cuando contenía calorías al ser mezclado con el azúcar. Este resultado está en desacuerdo con los reportes realizados por Mehiel y Bolles (1988). Mehiel y Bolles expusieron a ratas a diferentes sustancias de diversos sabores pero con igual contenido calórico. Las ratas mostraron preferencia por el sabor asociado con el etanol, que es una sustancia con alto contenido calórico y cuyo sabor desagrada a las ratas. También contradice los reportes experimentales que señalan que el sabor y el contenido calórico del azúcar son elementos facilitadotes en la producción de preferencias por sabores específicos, incluso por aquellos que son considerados aversivos (Capaldi, 1996).

Los resultados obtenidos en nuestro experimento parecen estar también en desacuerdo con los resultados obtenidos por Forestell y LoLordo (2000), debido a que indican que el sabor es un factor determinante en la elección y consumo de la comida, incluso por encima del contenido calórico o nutricio del mismo, o las necesidades de supervivencia del individuo. Esto puede ser debido a que las ratas y otros roedores han creado estrategias conductuales para evaluar el contenido nutritivo y de toxicidad de la comida basándose en ciertas señales, como lo es el sabor. El uso de esas señales es crítico para las ratas, ya que se encuentran desprovistas de la capacidad de regurgitar la comida una vez tragada (Aubert, 2005). Estas estrategias utilizadas en la elección de la comida incluyen: vacilación ante alimentos desconocidos, preferencia por los alimentos familiares, una especial habilidad para adquirir información sobre las consecuencias metabólicas tanto positivas como negativas de diferentes alimentos, y una preferencia innata por el dulce y en contraparte una aversión hacia los sabores amargos (Aubert, 2005).

Cabe mencionar que las ratas en este estudio consumieron la mezcla, aunque no de manera suficiente para cubrir sus necesidades energéticas, sobre todo durante el período de privación de comida. Este hallazgo parece estar de acuerdo con los resultados obtenidos por Aubert (2005). Aubert provocó un estado de enfermedad en ratas experimentales y a continuación les presentó soluciones de agua con quinina y con sacarina. Los resultados indican que pese al estado de enfermedad en que se encontraban los sujetos, éstos lograron discriminar el sabor dulce del amargo, y preferir la solución de agua con sacarina sobre la de agua con quinina, aunque también consumieron la solución amarga. Estos resultados sugieren que no se da un estricto rechazo de lo amargo en los roedores bajo ciertas circunstancias, como la enfermedad o la disponibilidad de la comida. 
Un fenómeno que es importante señalar son las grandes bebidas y comilonas registradas después de los periodos en que se proporcionó la mezcla glucosa-quinina, la quinina o se privó de comida. La ocurrencia de este tipo de comportamiento se ha reportado después de aplicar periodos de privación de agua o comida, totales o parciales (López-Espinoza, 2001, 2004). Sin embargo, solo las grandes bebidas y comilonas registradas en el último período de libre acceso, estarían relacionadas con la privación de comida aplicada durante la exposición a la mezcla glucosa-quinina con privación de comida; mientras que las registradas durante los períodos de línea base posteriores a la exposición a la glucosa, a la quinina y a la mezcla glucosa-quinina sin privación de comida estarían relacionadas con el sabor de estas sustancias. A partir de este resultado es posible afirmar que la ocurrencia de las grandes bebidas y comilonas también se encuentra relacionada con el sabor de la comida disponible.

Esta afirmación también es sustentada por las grandes bebidas registradas durante la exposición a la glucosa. Martínez, (2005) demostró que una fuente alterna de calorías en el agua modifica el patrón de consumo de agua y comida, produciendo grandes bebidas en los períodos en que se encuentra disponible la glucosa o posteriores a la privación de agua sin tener efecto sobre el peso corporal.

Los resultados obtenidos permiten concluir que el sabor es un factor determinante para el consumo de la comida. El efecto que el sabor aversivo tiene sobre el consumo de comida es contundente, se puede llegar hasta la severa autoprivación, que a su vez ocasiona la ocurrencia de grandes bebidas y comilonas. Sin embargo, es necesario diseñar nuevos experimentos que evalúen el efecto de diferentes concentraciones de sabor sobre la conducta alimentaria y el peso corporal

\section{REFERENCIAS}

Aubert, A. (2005). The taste of sickness: lipopolysaccharide-induced finickiness in rats. Physiology \& Behavior, 84, 437-444.

Bartoshuk, L. (1990). Distinctions between taste and smell relevant to the role of experience. En E. Capaldi y T. L. Powley (eds.). Taste, experience \& feeding: Development and learning. USA: American Psychological Association, 62-72.

Booth, D. (1990). Learned role of tastes in eating motivation. En E. D. Capaldi, y T. L. Powley (eds.). Taste, experience \& feeding: Development and learning. USA: American Psychological Association, 179-194.

Capaldi, E. D. (1996). Conditioned Food preferences. En E. D. Capaldi (ed). Why we eat, What we eat. USA: American Psychological Association,53-80.

Del Prete, E. (1994). Meal pattern of rats during hyperphagia induced by long-term food restriction is affected by diet composition. Appetite, 23, 79-86. 
Fedorchak, P. M., y Bolles, R. C. (1987). Hunger enhances the expression of caloriebut not taste-mediated conditioned flavor preferences. Journal of Experimental Psychology: Animal Behavior Proceses, 13, 1, 73-79.

Finger, F. W. (1951). The effect of food deprivation and subsequent satiation upon general activity in rat. Journal of Comparative and Physiological Psychology, 44, 557-564.

Forestell, C. A., y LoLordo, V. M. (2000). Can orally consumed calories condition preferences for relatively unacceptable tastes?. Learning and Motivation, 31, 153179.

López-Espinoza, A. (2001). Efectos de la privación de agua y comida sobre el peso corporal y el consumo de alimento y agua en ratas albinas (Rattus norvegicus). Tesis de maestría inédita, Universidad de Guadalajara, Jalisco, México.

López-Espinoza, A. (2004). Análisis experimental de los efectos post-privación. Una propuesta para el control de la gran comilona en ratas albinas (Rattus norvegicus). Tesis doctoral inédita, Universidad de Guadalajara, Jalisco, México.

López-Espinoza, A. y Martínez, H. (2001). Efectos de dos programas de privación parcial sobre el peso corporal y el consumo total de agua y comida en ratas. Acta Comportamentalia, 9, 5-17.

Martínez, A. G. (2005). Efectos diferenciales de la glucosa sobre el peso corporal, consumo de alimento, agua y calorías durante el período post-privación en ratas albinas (Rattus norvegicus). Tesis de maestría inédita, Universidad de Guadalajara, Jalisco, México.

Mason, D. J. (1965). Palatability of sugar of lead. Journal of Comparative Physiological Psychology, 59, 1, 94-97.

Mehiel, R., y Bolles, R. C. (1988). Learned flavor preferences based on calories are independent of initial hedonic value. Animal Learning \& Behavior, 16, 383-387.

Mennella J. A., y Beauchamp, G. K. (1996). The early development of human flavor preferences. En E. D. Capaldi (ed). Why we eat, What we eat. USA: American Psychological Association, 83-112.

Rozin, P. (1965). Specific hunger for thiamine: recovery from deficiency and thiamine preference. Journal of comparative and physiological psychology, 59, 1, 98-101.

Schafe, G. E. (1996). Taste aversion learning. En E. D. Capaldi (ed). Why we eat, What we eat. USA: American Psychological Association, 31-51.

Sclafani, A. y Gorman, A. N. (1977). Effects of age, sex, and prior body weight on the development of dietary obesity in adult rats. Physiology \&Behavior, 18, 10211026.

Sclafani, A. (1990). Nutritionally based learned flavor preferences in rats. En E. D. Capaldi y T. L. Powley (eds.). Taste, experience \& feeding: development and learning. USA: American Psychological Association, 139-156.

Subcommittee on Laboratory Animal Nutrition, Committee of Animal Nutrition, Board on Agriculture, National Research Council. (1995). Nutrient Requirements of Laboratory En línea : National Academies Press. 